\title{
MiNI-IMPLANTS IN ORTHODONTICS
}

\author{
SZUHANEK C. \& POPA A.
}

Abstract: The orthodontic mini-implants are a new and developing clinical tool. Many questions are still unanswered while large numbers of biological science and clinical research papers already provide a sound evidence base. It has been proven that miniimplants are minimally invasive, safe, well tolerated, cost effective and provide absolute anchorage. Studies have been conducted trying to develop a simple protocol for mini-implant insertion, increase the success rate and to easily introduce skeletal anchorage in the clinical practice. Many variables affect the primary stability of miniimplants: cortical bone thickness, insertion technique, loading protocols, mini-implant design and root proximity. To increase the success rate for the mini-implants precise insertion has to be provided. Surgical guides have been fabricated on models replicated with CBCT.

Key words: mini-implant, surgical guide, CBCT, orthodontics, biomechanics
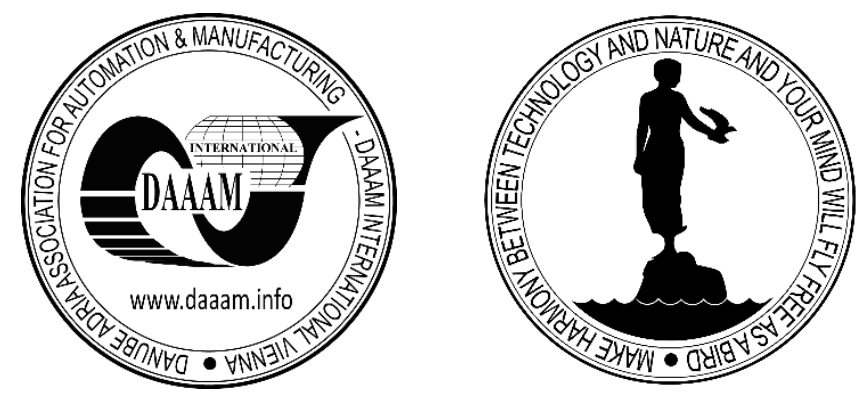

Authors' data: S.L. Dr. Szuhanek, C[amelia], Dr. Popa, A[delina], Faculty Of Dental Medicine, University Of Medicine And Pharmacy "Victor Babeș" Timișoara, România, PiațaEftimieMurgu nr. 2, Timișoara, România, cameliaszuhanek@umft.ro, adelina88_popa@yahoo.com

This Publication has to be referred as: Szuhanek, C[amelia] \& Popa, A[delina] (2016). Mini-Implants in Orthodontics, Chapter 28 in DAAAM International Scientific Book 2016, pp.315-324, B. Katalinic (Ed.), Published by DAAAM International, ISBN 978-3-902734-09-9, ISSN 1726-9687, Vienna, Austria

DOI: $10.2507 /$ daaam.scibook.2016.28 


\section{Introduction}

The concept of skeletal anchorage was first introduced more than 70 years ago when Gainsforth and Higley inserted Vitallium screw in dogs concluding that anchorage may be obtained for orthodontic movement in the future [Gainsforth B L , Higley L B, 1945]

Since then many studies have been conducted trying to develop a simple protocol for mini-implant insertion, increase the success rate and to easily introduce skeletal anchorage in the clinical practice.

\section{Terminology}

A large variety of terms have been used to describe the orthodontic mini-implant mini-screw [Dalstra, 2004], mini-implant [Hong, 2004], micro-implant [Chung, 2005], micro-screw [Park, 2003]. The word "micro" is short from microscopic so the terms micro-implant or micro-screw are not suitable because these appliances are small (mini ) not microscopic.

The term osseointegration and mini -implants is also ambiguous. Osseointegration is defined as a state in which, under the optical microscope, there is direct contact between the implant and bone without any intervening soft tissue, and which enables transmission of the external stresses to the bone structure in a functional manner [Szuhanek 2009, 2015].

Mini-implants are mechanically retained by the interlocking between the screw and bone rather than forming a clinically discernible ankylotic union with the bone.

\section{Classification}

1. According to the shape and size

a) Conical (cylindrical)

b) Mini-plate implants

c) Disc implants (onplants)

2. According to implant bone contact

a) Osteointegrated

b) Non-osteointegrated

3. According to the material

a) Biotolerant

- Stainless steel

- Chromium-cobalt alloy

b) Bioinert

- Titanium

- Carbon

c) Bioactive

- Hydroxyapatite

- Ceramic oxidized aluminium 
4. According to the application

a) Used only for orthodontic purposes

b) Used for prosthodontics and orthodontic purposes

\section{Concepts and controversies}

\subsection{Method of insertion: Drill-free or pre-drilling}

In the drill-free method, the mini-implant is directly inserted into the placement site without the need of a pilot hole. In the pre-drilling method, the mini-implant is inserted into a guide-hole drilled before its placement.

Gupta et al evaluated the stability of self-tapping and self-drilling screws. They demonstrated that both the self-tapping and the self-drilling screws were effective anchorage units. They described the advantages of self-drilling screws, which included decreased operative time, little bone debris, less thermal damage, lower morbidity, and minimal patient discomfort because predrilling is not required. If both the self-tapping and the self-drilling methods result in placement with equal stability, then the selfdrilling method should be preferred because of its clinical advantages. [Gupta N , 2012]

Pre-drilling technique has some disadvantages, specially for interradicular areas: damage to adjacent tooth roots, drill-bit break-age, thermal necrosis of the surrounding bone that contributes to the implants loss of stability [Heidemann W , 1999]

Park et al stated that self-drilling screws are not recommended for placement in dense and thick cortical bone such as the mandibular molar region, the self-tapping method is preferred to prevent fracture of the screw or the bone [Park HS, 2008]

\subsection{Timing of loading: immediate versus delayed loading}

Many clinicians have shown that the mini-implant can be successfully loaded without having to wait for several months. Melsen et al performed a histological evaluation of the bone-screw contact after 1,3 and 6 months intervals prior to loading based on which they advocated immediate loading [Melsen B. 2005]. Park stated that it is possible to apply orthodontic force once the soft tissues have healed [Park H 1999]. It is important to load a low initial force, less than $50 \mathrm{cN}(50 \mathrm{~g})$, if it is applied soon after mini-implant placement. A screw can loosen as a result of application of a force that exceeds the amount that can cause microfractures in the thin cortical bone. [Melsen B 2005] This is easily applied by a elastomeric chain and it allows time for the bone healing response to stabilize the mini-implant. Indirect anchorage loading may also be applied immediately.

\section{Indications, counterindications}

There are two basic forms of absolute anchorage [Echarri, 2007]

a) Direct anchorage : when active segment is pulled directly from the mini-implant

b) Indirect anchorage : when active segment is pulled from the reactive segment, and this segment is fixed to mini-implant to increase anchorage 


\section{Factors that influence the success rate}

Many studies have been conducted to evaluate the risk factors for failure of miniimplants to improve the success rate [Deguchi, 2006]. They found that the primary stability of mini-implants is related to the mechanical characteristics of the interface between the mini-implant and bone in relation to factors such as bone quality and quantity, and screw diameter, length and design. [Miyawaki, 2003]

According to Raed Alrbata: The single most important factor for the success of the mini-implant is bone

\subsection{Mini-implant design factors}

The diameter and thread length of the mini-implant are the main characteristics to consider when selecting a mini-implant. Most of the current mini-implants are of the drill-free or self-drilling type and have a $1.6 \mathrm{~mm}$ diameter. This $1.6 \mathrm{~mm}$ diameter is considered to have sufficient rigidity to be inserted without drilling The drill-free mini-implants have a cutting flute that allows insertion without drilling. [Cheol-Ho, 2009]

The drill-free mini-implants come in a variety of thread lengths $(5-9 \mathrm{~mm})$. They are available in two configurations: cylindrical with a diameter of $1.6 \mathrm{~mm}$ and tapered with a maximum diameter of $1.6 \mathrm{~mm}$ or $1.8 \mathrm{~mm}$. There are available even longer implants $>11 \mathrm{~mm}$. The length to be used depends on the thickness of soft tissue and cortical bone at the site of placement. In the midpalatal area the use of shorter miniimplants is suggested $(5 \mathrm{~mm})$. In the buccal alveolar area to achieve maximum contact with the cortical bone, $6 \mathrm{~mm}$ length is recommended. Longer mini-implants are recommended in the retromolararea $(>8 \mathrm{~mm})$ and palatal alveolar regions $(>7 \mathrm{~mm})$ [Cheol-Ho, 2009]

\subsection{Insertion technique}

Accurate mini-implant positioning provides retention during orthodontic loading and precise control of the force vector, reduces problems such as loosening of the implant or invasion of the sinuses, periodontal ligament, root surface. The use of a surgical guide facilitates reliable placement of the implant [Kravits, 2007, Stratul 2010 Serban 2009].

If the placement of the mini-implant relies on only the clinician's technique, skill, and experience, complications can occur. Root contact or damage, penetration of the maxillary sinus, and insufficient bone area for the mini-implant due to alveolar bone loss. Under these circumstances, the screw can become loose. [Maino , 2005]

When the mini-implant has to be placed near delicate anatomical structures: roots maxillary sinus, alveolar nerve, a surgical guide has to be used to precisely locate the placement point.

There have been various studies to standardize the proper positioning of miniimplants. The placement site is critical to ensure a successful outcome, but the more important point is the implant guide.

The traditional surgical guide (Figure 1 ) is manufactured using 2- dimensional images that do not allow accurate view of the area, so errors can occur during insertion. 
Bae etal reported on a guide wire that provides a reference in the $\mathrm{X}$-rays.[Bae ,2002 ].Maino et al used resin guides and several $\mathrm{x}$-rays with the long-cone parallel method, but this approach is technique sensitive [Maino, 2005].

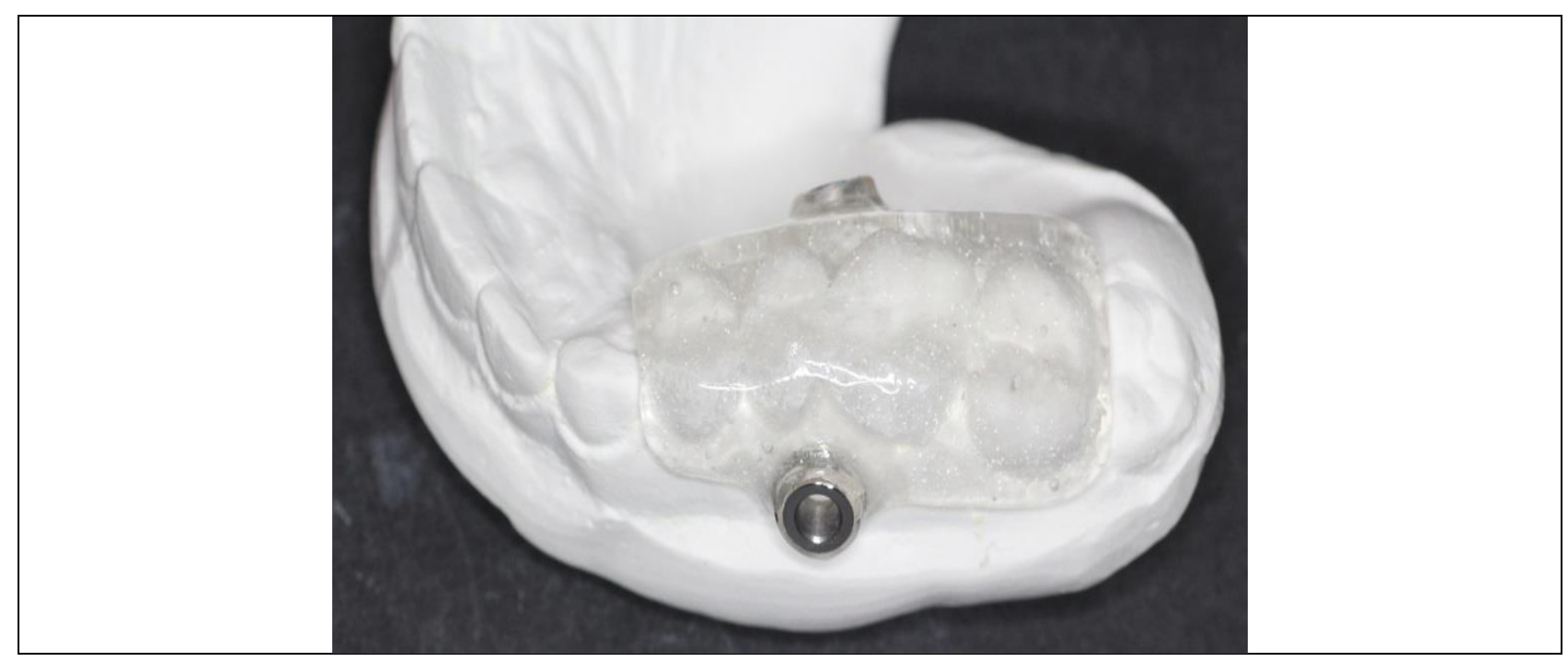

Fig. 1. Resin surgical guide

To overcome this drawback a more precise surgical guide was developed using CBCT replica model. The clinical application of CBCT in fabricating a surgical guide allows for more accurate and safe placement of mini-implants in the interradicular spaces of the alveolar area [Yu JJ, 2012] The technique produces a reliable surgical guide but the complexity of the fabrication procedure, extensive laboratory work and high cost limit the practical use of the system. [Seong-Hun 2008]

\section{Clinical procedure}

Studies indicate that the drill-free mini-implants provide extensive implant-bone contact, with little bone debris and less thermal damage than pre-drilling screws.[Kim 2005] The most commonly used mini-implant is $1,6 \mathrm{~mm}$ diameter and is considered to have sufficient rigidity to be inserted without drilling.

\section{Armamentarium}

\section{Hand instruments}

- Straight hand driver (fig 2)

- Short hand driver

- Contra-angle hand driver

\section{Motor-driven rotary instruments}

- Implant motor with attached hand-piece

- Low-speed hand-piece with contra-angle head run

- Connecting burs

- Pilot drills 


\section{Orthodontic mini-implants (Fig 3)}

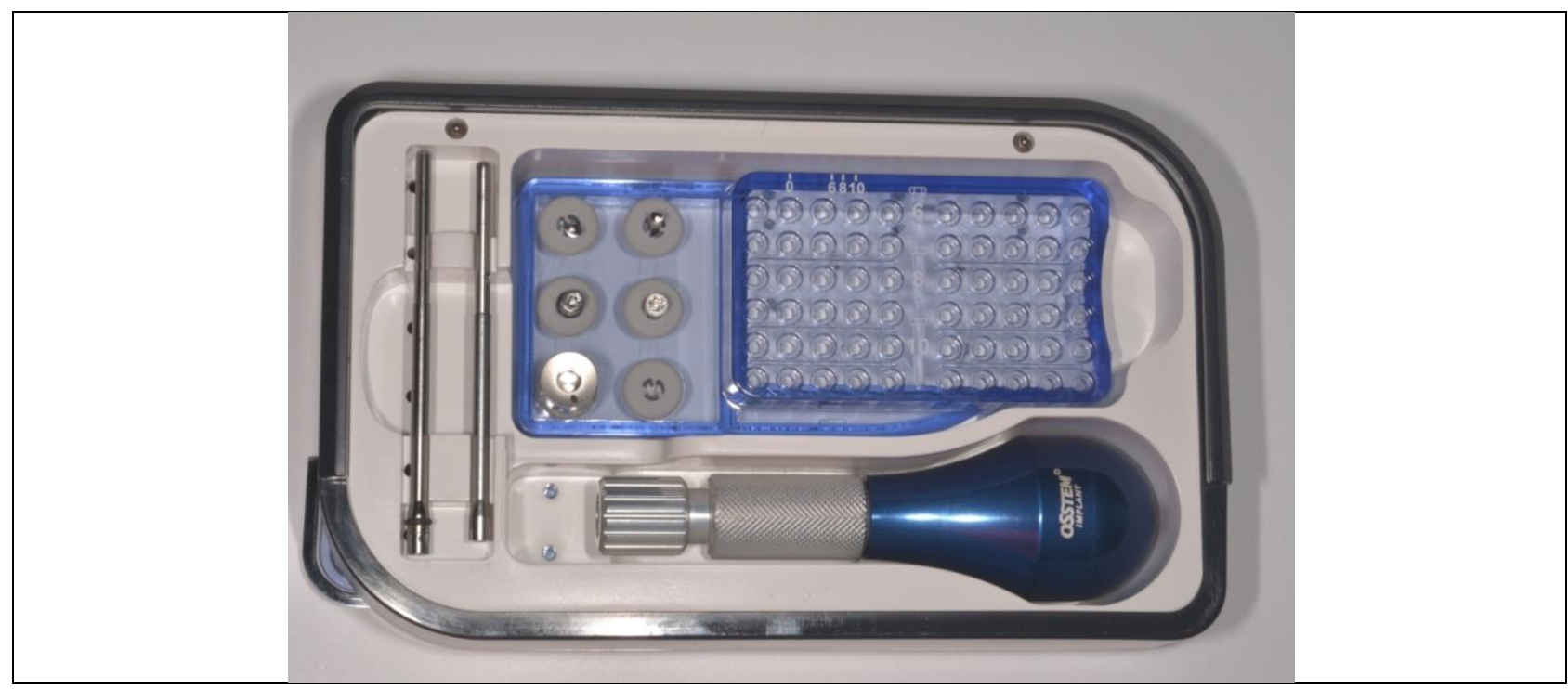

Fig. 2. Mini-implant kit - Osstem

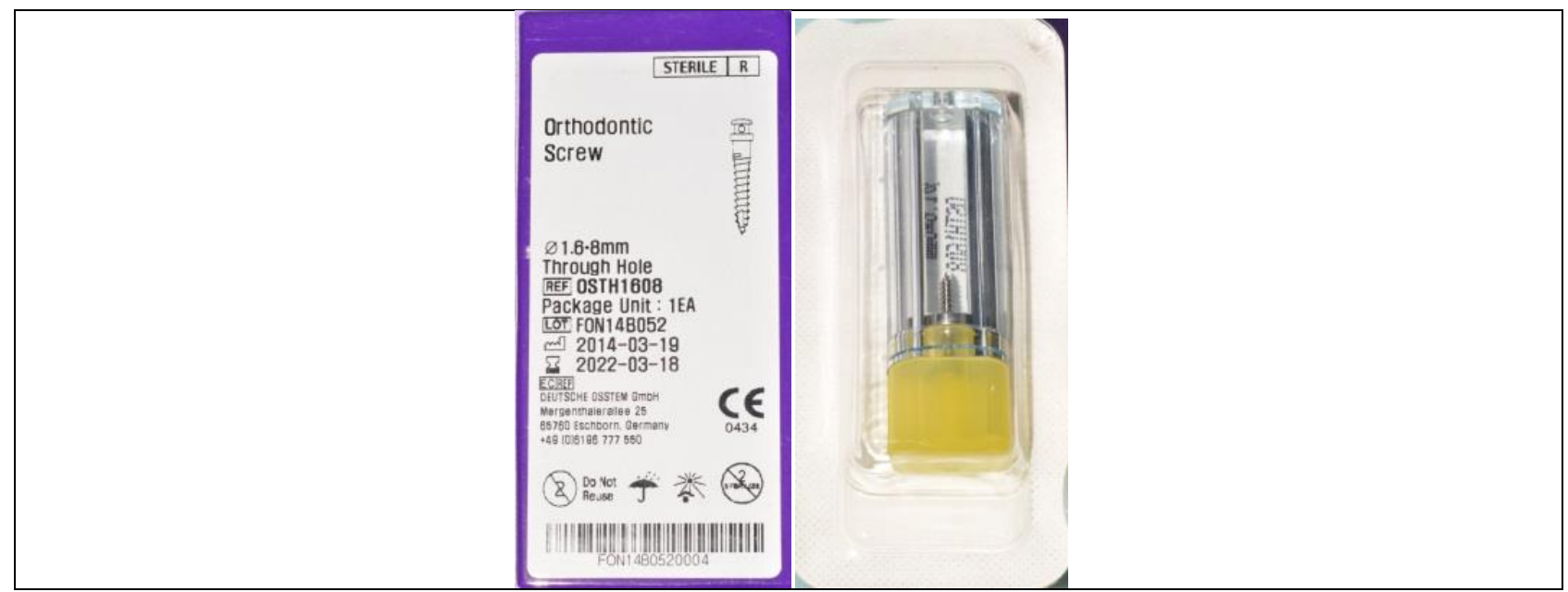

Fig. 3. Mini-implant :Osstem (1.6 x 8mm)

\section{Anatomic considerations}

Factors that need to be considered when choosing a site for the mini-implant placement: anatomic structures in the vicinity of the site of placement, bone quality, soft tissue thickness, accessibility, usability, patient comfort.

\section{Anatomic structures in the vicinity of the site of placement}

\section{Bone quality}

To provide good stability for the mini-implant the quality and quantity of the cortical bone is essential. The thickness and density of the bone varies between different anatomic sites.

The mandible provides more buccal cortical bone than the maxilla [Yun H S 2001]. The mandibular buccal region has the thickest cortical bone of all regions 
evaluated [Farnsworth, 2011]. The cortical bone in the mandibular buccal region is thicker than the cortical bone in the maxillary buccal and lingual region. Cortical bone thickness in the mandibular buccal regions increases from anterior to posterior as reported by Baumgaertel [Sebastian Baumgaertelaand 2008, Popa 2015].

The maxillary cortical bone is thicker in the palate than on the buccal surface [Yun $\mathrm{H}$ S 2001].

\section{Soft tissue thickness}

When determining the length of the mini-implants, the soft tissue thickness has to be taken into consideration.

The soft tissue on the palatal site is thicker than on the maxillary bucalsite. In the palate, soft tissue thickness is greater between the first and second molars [Yun H-S, 2001]. The midpalatal region has good soft tissue characteristics for mini-implant placement along with bone quality. Along the midpalatal suture, the mucosa is thickest at the area $4 \mathrm{~mm}$ distal to the incisive papilla and the rest of the posterior area has a uniform soft tissue thickness of $1 \mathrm{~mm}$ [Yun H-S, 2001].

\section{Usability}

The mini-implant has to be placed in a favorable biomechanical site to allow for the application of the orthodontic force [Yun H-S 2001]. The position of the mini-implant has to allow the system to be adjusted along with the treatment progress

\section{Placement procedure}

The orthodontic mini-implant will be inserted using a straight hand driver. A connection bur is mounted on the hand piece to connect the hand piece with the miniscrew (Figure 4,5).

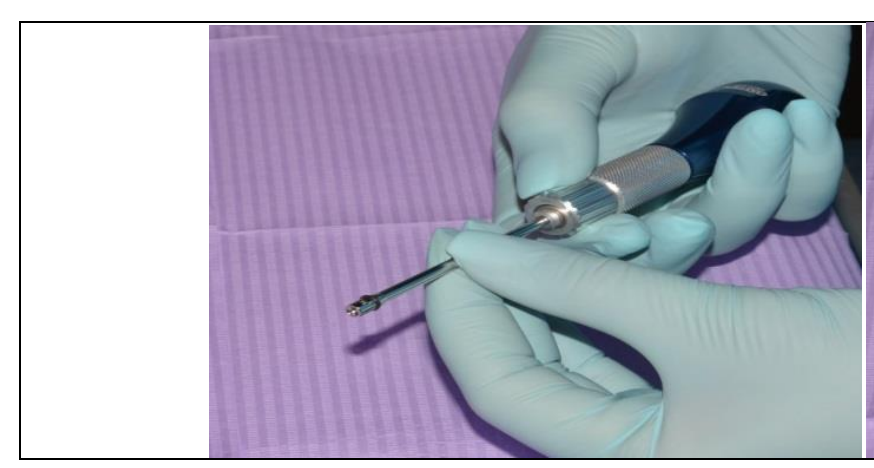

Fig. 4. Mounting the connection bur

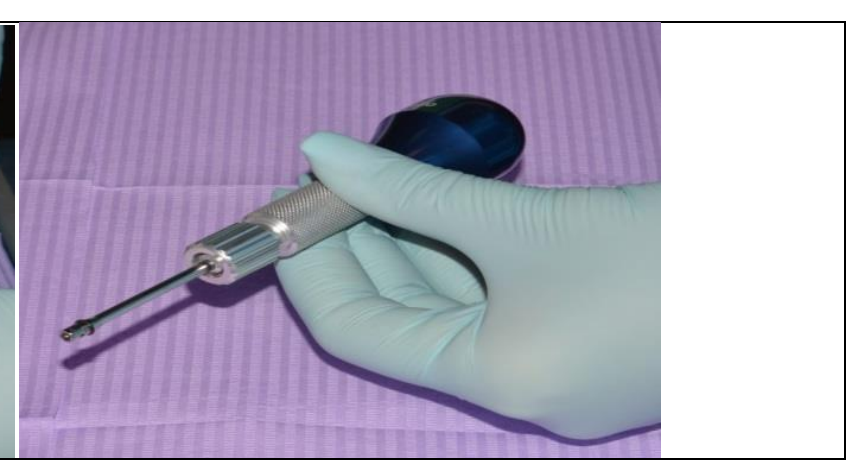

Fig. 5 Handpiece with connection bur

When the mini-implant is connected to the hand driver (Figure 6,7 ) the core of the mini-implant should not come in contact with other than sterilized instruments. 


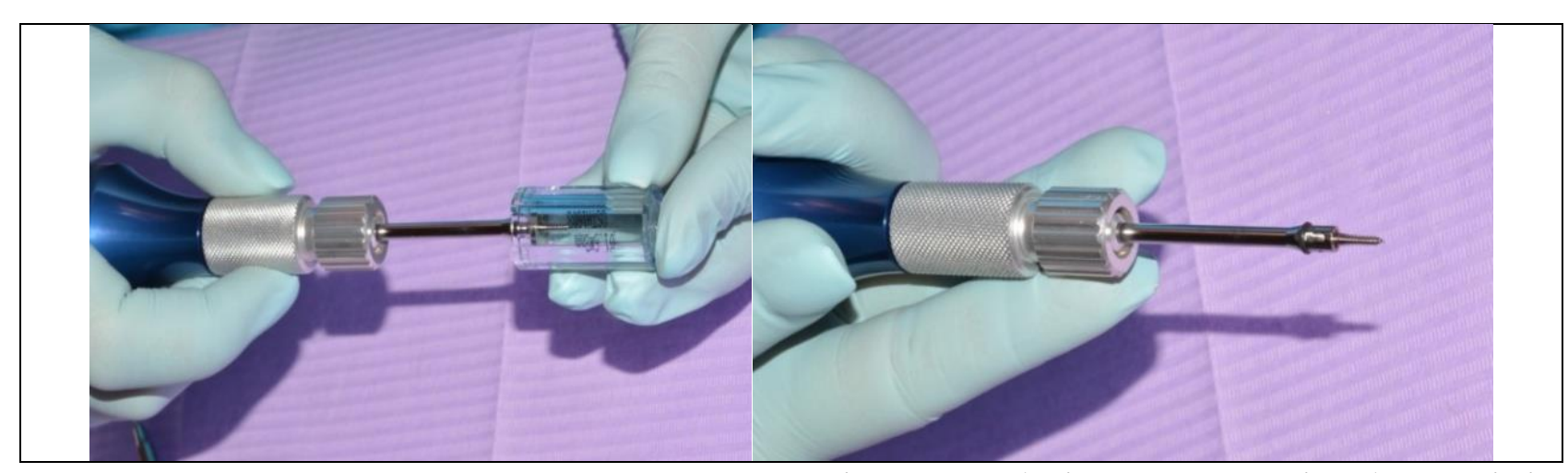

Fig.6. Mini-screw pick up

Fig.7. Hand piece- connection bur- mini implant

Maxillary buccal alveolar area - using straight hand driver

1. Determine the site before insertion by placing the parodontal probe parallel to the long axis of the teeth. Appropriate height is determined by viewing the panoramic radiograph or CBCT investigation. The mini-screw has to be placed in the attached gingiva .(Figure 7 )

2. A pinpoint mark is made at the planned insetion site with the probe . (Figure 8)

3. The miniscrew is mounted on a hand driver and secured on the cortical bone surface, before driving throught the bone ( Figure 9 )

4. Drive in the screw by rotating the hand driver clockwise at less than $30 \mathrm{rtm}$ ( $1 / 4$ rotation per second )

5. Stop driving when the head of the screw lies at the level of the surface of the gingiva .( Figure 10)

6. Post placement instructions for the patient: The precautions are explained to the patient. The patient may experience pain for 1 or 2 days, so they can take analgesics if required. Aspirin is not recommended because it has been reported to inhibit tooth movement. The brushing is gentle not tapping the mini-implant head with the toothbrush.

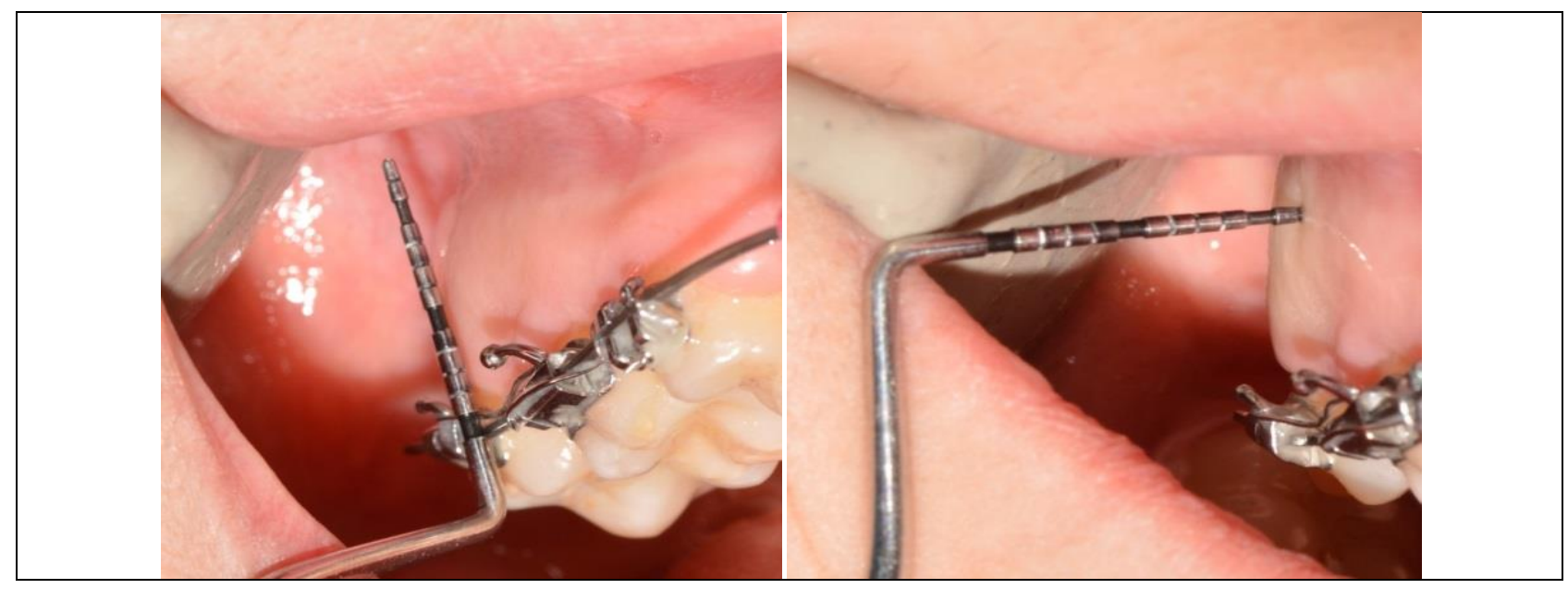

Fig.8. Determine the insertion site

Fig. 9. Mark the insertion site 


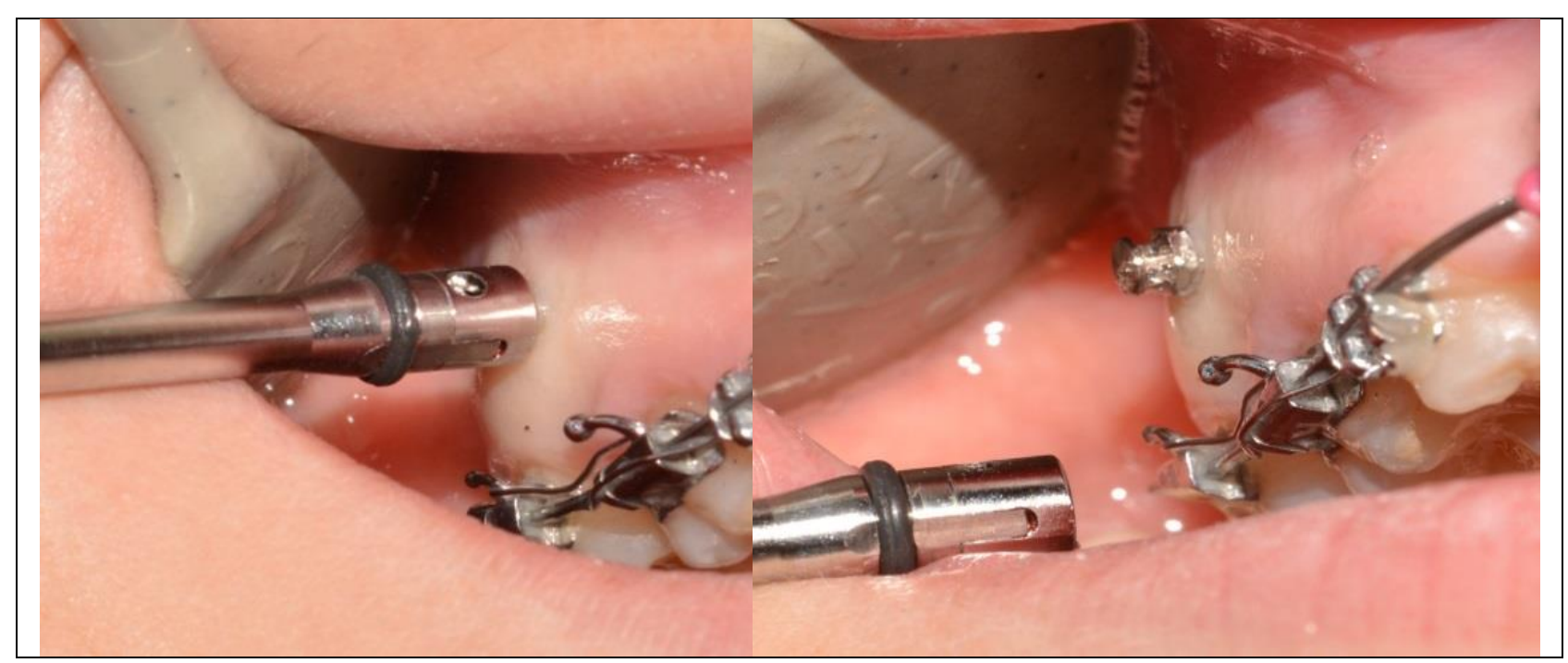

Fig. 10. Mini-implant insertion

Fig.11. Inserted mini-implant

Complications may include: damage to anatomic structures, lack of primary stability, high resistance to insertion, deflection of the insertion path, soft tissue problems, patient discomfort, mobility of the mini-implant, poor oral hygiene and inflammation [Cheol-Ho P et al 2009, Kamlesh 2010].

\section{Conclusion}

The introduction of mini-implants in orthodontic therapy had eliminated the previous biomechanical barriers. Nowadays, due to the advanced technology in orthodontics, adut patients may benefit from various types of treatment that will enable the interdisciplinary approach and will achieve a better clinical result.

\section{References}

Cheol-Ho P et al . Orthodontic mini-screw implants 2009. 24-25

Chung K, Kim S H, Kook Y C 2005. Orthodontic microimplant for distalization of mandibular dentition in class III correction. Angle Orthodontist 75:119-128

Dalstra M , Cattaneo P M , Melsen B 2004 Load transfer of miniscrew for orthodontic anchorage. Orthodontics 1:53-62.

Gainsforth B L, Higley L B 1945. A study of orthodontic anchorage possibilities in basal bone. American Journal of Orthodontists and Oral Surgery 31 :406-416

Ghiban, B., Bortun, C.M., Faur, N., Ghiban, N., Cernescu, A., Ghiban, A. and Serban, N., 2009. Mechanical behaviour of composite resins for dental applications. Annals of DAAAM \& Proceedings, pp.773-775.

Hong R K, Heo J M, Ha Y K 2004. Lever arm and mini-implant system for anterior torque control during retraction in lingual orthodontic treatment. Angle Orthodontist 75:129-141

Melsen B, Verna C 2005. Miniscrew implants: the Aarhus anchorage system. Seminars in Orthodontics 11:24-31 
Melsen B 2005. Mini-implants: Where are we? Journal of Clinical Orthodontics 39:539-547

Park H S 1999. The skeletal cortical anchorage using titanium microscrew implants. Korean Journal of Orthodontics 29:699-706

Park H 2003 Clinical study on success rate of microscrew implants for orthodontic anchorage. Korea Journal of Orthodontics 33:151-156

Popa A., Szuhanek C., Brad Silviu - Accurate Determination for Orthodontic Miniimplant Placement Using Acrylic Resin Surgical Guide and CBCT.Revista Materiale plastice, vol. 53, nr. 2, 2016, Bucuresti. ISSN 0025-5289

Stratul, Stefan Ioan, et al. Thermo graphical methods in highlighting the muscle strain of dentists during dental treatments under the microscope.Annals of DAAAM \& Proceedings (2010): 1365

Szuhanek Camelia. Material characteristics of the orthodontic archwires. volumul DAAAM International Scientific Book 2011Published by DAAAM International, Vienna, Austria. decembrie 2011 DOI: 10.2507/daaam.scibook.2011.xx.ISBN 978-3901509-84-1, ISSN 1726-9687

Szuhanek C., Grigore A.- Determination of microelements from orthodontic implans by the flame atomic absorption spectroscopy method. Revista de Chimie. Vol 10/2015. ISSN: 0034-7752

Szuhanek C., Faur N., Cernescu A.: Biomechanical 3D analysis of stress induced by orthodontic implants. Key Engineering Materials, vol.399(2009), pp 194-204

Szuhanek C., Cernescu A., Faur N., Glavan F., Fleser T., Vatau S. - Finite element simulation of first molar movement during orthodontic treatment. Annals of DAAAM for 2009 Proceedings vol.20 no.1 pag.953-954. ISBN 978 - 3- 901509-70-5

Sebastian Baumgaertelaand Mark G. Hans: 2008 Buccal cortical bone thickness for mini-implant placement. AJODO

Yun H S 2001 The thickness of the maxillary soft tissue and cortical bone related with an orthodontic implantation [master's thesis]. Seoul, South Korea: Yonsei University. Kamlesh S 2010 Temporary anchorage devices - Mini-implants , Natl J Maxillofac Surg. 2010 Jan-Jun; 1(1): 30-34 